\title{
Animal research and the search for understanding
}

$t$ is all too easy for the public to underestimate how little understanding and control humans have over biology and how limited and contingent our greatest advances in medical science have been. Researchers must continually remind the public that animal experimentation is essential, not only for its clinical applications to human and animal health, but because it is usually the only feasible way to examine basic questions of structure, function, development, behavior and even welfare. It is significantly more difficult to do this effectively if we are required to treat animal experimentation as a moral imperfection to be eliminated.

The full range of opinions found in society about subjecting nonhuman animals to experiments is mirrored among scientists themselves. In recognition of this, a comprehensive report, entitled 'The Ethics of Research Involving Animals', by the Nuffield Council on Bioethics (http://www.nuffieldbioethics.org/) grounds its cogent arguments in not one, but a series of ethical standpoints, ranging from 'anything goes' to an abolitionist stance. Its conclusions are designed to reinforce the UK Home Office regulations for animal experimentation, which in 1959 introduced the '3R' goals-refinement, reduction and replacement-ultimately treating animal experimentation as a problem requiring regulatory reduction. The report also aims to open constructive dialogue with those who might otherwise sympathize with the UK's uniquely awful 'animal rights' terrorists (http://www.economist.com/world/europe/ displayStory.cfm?story_id=4316824).

This dialogue is desirable if it helps to deter vandalism of scientific institutions and attacks on scientists themselves.

An ethical framework is an essential tool with which to train scientists to question and explain the significance of their research. It can also spur consistent implementation of best practice and can shape legislation. Coupled with the highest professional standards of conduct and regular public communication, it helps keep researchers in touch with the range of public views regarding their experiments and attitudes. These attitudes vary locally across the world, but the UK has set an excellent precedent: establishing the right ethical code creates a local advantage that greatly promotes research. The preemptive studies and establishment of regulatory guidelines by the UK Human Fertilisation and Embryology Authority allowed the nation to gain an edge in the fields of in vitro fertilization and stem cell research within an ethical paradigm (http://www.hfea.gov.uk/Home).

Has the UK also established the right principles for animal work? The case for 'refinement, reduction and replacement' of animal research has been the guiding principle of government licensing since 1959 and is now embodied in a national organization, NC3Rs (http://www.nc3rs.org.uk/), supported by the government, the largest funding bodies and the Association of the British Pharmaceutical Industry. Many UK researchers themselves start from this position (http://www.rds-online.org.uk). In contrast, at least six of the organizations represented by the UK's Association of Medical Research Charities (http://www.amrc.org.uk/) make no explicit mention of the principles under which licensing is carried out for the animal research that they fund. Instead, they argue, disease by disease, the case for why the insights gained from animal experiments are needed to deliver advances in human and animal health and welfare.

To our knowledge, in the US, only the American Veterinary Medical Association, the Society of Toxicology and the Howard Hughes Medical Institute explicitly promote the '3R' slogan. At least ten of the professional biomedical research associations whose public policy statements we examined (http://www.fbresearch.org/Education/ index.htm\#Opinions) explain the importance of animal research in terms of basic understanding and expected health benefits. All do so within an ethical framework that includes appropriate training, legislative oversight, humane treatment of animal research subjects and minimization of animal distress and suffering.

Our ethical position is that as humans, researchers have a unique opportunity to obtain understanding of the world. Their position of control brings the professional responsibility to use animals humanely, to justify why they are used, and to explain publicly exactly what is being done. If this does not persuade the public in one country, the work must be done in another, where the benefits of the knowledge gained will naturally accrue. Researchers are already mobile in their careers, and some will readily relocate to more supportive environments. Their need to maintain a reputation demands that the research be ethically supported. As with other challenging research, this is an invitation to the host nation to mandate and fully fund best practice in education, oversight and legislation. The global research community of peer referees and the global public with access to the research results will not long tolerate national governments that condone poor welfare practices or cruelty. As stressed or crowded animals produce unreliable research results, and many phenotypes are only accessible in contented animals in enriched environments, it is in the best interests of the researchers not to cut corners or to neglect welfare issues. The animals, too, have their own interests, and these must be taken into account when experimenting upon them. Because they have only the rights afforded to them by people, this is our responsibility.

It is all too easy to see that refinement (bigger cages, enriched environments) will cost more money, while reduction might save money. Research into replacing animals has now given rise to 
high-profile institutes in their own right (see http://www.vetmed. ucdavis.edu/Animal_Alternatives/main.htm and http://caat.jhsph. edu/, for example). While it is important to ensure the robustness of research findings through other experimental approaches, we do not favor the use of extra animals simply to establish alternative methodologies. Together, these principles imply phasing out animal research. This, in turn, may mean a progressive reduction in funding, which, in turn, might increase the pressure on conditions with the potential to affect welfare. It may well be that the least restrictive o regulatory environments encourage development of the healthiest and best-funded animal facilities, and stringent regulatory requirements result in delays and underfunded conditions, which impose their own welfare costs.

Declaring the intention to phase out animal research undermines the argument for its importance to our understanding and control of the natural world. If a researcher holds both positions simultaneously, opponents have only to point to inconsistencies between principle and practice to begin to cast doubt on the honesty of the scientists. From there, it is a short step to impugn their reputations and the validity of their research results. No wonder so few exercise their duty to explain, and public fears of secrecy go unallayed. There is no such conflict in advocating the use of animals in welfare research, which we do wholeheartedly. Several excellent centers for welfare research are pioneering the scientific investigation of all the consequences of animal use, not only in laboratories but also in the broader context of animals bred for food, hides, pelts and pets, across the range of conditions animals experience in the wild and under pest-control programs (see http://www.faifarms.co.uk/ and http:// www.uoguelph.ca/abw/index.shtml, for example).

Genetic alteration and reproductive cloning offer powerful new insights, mostly in rodents. Some 300,000 genetic lines of mice are envisaged, several million mice per year are used for phenotyping in the UK alone, and many animals are needed to propagate the experimental animals. The Nuffield report recommends that complete phenotypes of studied animals (including welfare phenotypes and new genetically modified animals, clones and mutants) should be recorded in database ontologies, and that NC3Rs should review welfare assessment systems and ontologies with a view to rationalizing them and examining their relationships. How would this work in practice? Imagine that ablation of a single gene in a mouse produces a syndrome comprising a developmental bone defect, a cancer predisposition and a behavioral abnormality. Each phenotype is likely to be gathered by thorough investigation in separate studies (and hence separate publications), and each has implications for the way in which experiments are designed and the way in which the animals are maintained and handled. Examples of phenotypes that may affect welfare would be if few of this strain live more than a year, or if they have impaired balance. Phenotypes are not simply recorded, but they depend upon the context of a particular investigation.

Within the agenda of reducing animal research, the expansion in genetically altered animals appears incongruous. Welfare phenotyping is, in effect, a ratchet mechanism supplied by the researchers themselves to invite regulators to put them out of business. The phenome database could create the unfortunate, incorrect impression - simply because this community has the funding and organization to pay proper attention to phenotyping - that genetically altered animals present qualitatively different welfare concerns from those presented by domestic animals bred in other situations.

In contrast, seen through our framework that animal research is key to understanding, complete phenotyping is essential to the collaborative progress in research that mouse and rat researchers have already initiated. Shared access to strains and experimental observations make research more effective by improving experimental design and reducing duplication of effort. Phenotypes relevant to welfare can be studied by welfare researchers, and all can make use of the information to progressively minimize distress and suffering in experimental animals. We agree with the Nuffield report that welfare information should be integral to the phenotyping in the databases rather than given exceptional status at a 'bioethics' site.

Understanding is still limiting. Without the voices of the scientists to give animal research its proper context, how is the public to understand its importance? We are certain that scientists are not well served by any attempt to protect them with a conflicted ethical framework that destroys public trust and ties their hands. 